\title{
Tunneling through an eternal traversable wormhole
}

\author{
Tian-Gang Zhou ${ }^{1}{ }^{1}$ and Pengfei Zhang $\mathbb{1}^{2, *}$ \\ ${ }^{1}$ Institute for Advanced Study, Tsinghua University, Beijing 100084, China \\ ${ }^{2}$ Institute for Quantum Information and Matter and Walter Burke Institute for Theoretical Physics, California Institute of Technology, \\ Pasadena, California 91125, USA
}

(Received 15 September 2020; accepted 2 December 2020; published 16 December 2020)

\begin{abstract}
The Maldacena-Qi model describes two copies of the Sachdev-Ye-Kitaev model coupled with an additional coupling and is dual to the Jackiw-Teitelboim gravity, which exhibits an eternal traversable wormhole in the low-temperature limit. In this work, we study an experimental consequence of the existence of the traversable wormhole by considering the tunneling spectroscopy for the Maldacena-Qi model. Making comparisons to the high-temperature black-hole phase where the bulk geometry is disconnected, we find that both the tunneling probability and the differential conductance in the low-temperature wormhole phase show nontrivial oscillation, which directly provides an unambiguous signature of the underlying SL(2) symmetry of the bulk geometry. We also perform bulk calculations in both high- and low-temperature phases, which match the results from the boundary quantum theory.
\end{abstract}

DOI: 10.1103/PhysRevB.102.224305

\section{INTRODUCTION}

Holographic duality has established many valuable connections between some strongly interacting quantum systems and the semiclassical gravity theory [1]. The Sachdev-YeKitaev (SYK) model [2-5], which describes $N$ randomly interacting Majorana zero modes, is one of the concrete examples where a holographic description exists. In the large- $N$ and low-temperature limit, the model effectively describes the Jackiw-Teitelboim gravity in two-dimensional nearly anti-de Sitter $\left(\mathrm{AdS}_{2}\right)$ space-time [6]. Later, several generalizations of the SYK model have been introduced to study different physics [7-22], including tunneling spectroscopy [7-10] for generalizations with $\mathrm{U}(1)$ symmetry [11-13]. Moreover, the quantum simulation of the SYK model [23] has been performed in NMR systems [24] and there are several other proposals for realizing the SYK model in different experimental systems [25-28].

Meanwhile, wormholes have become a central topic in the fields of gravity and quantum information. It is an essential entry point to understand the quantum teleportation $[29,30]$, the late-time behavior of the spectral form factor [31], and the resolution of the information paradox [32,33]. Besides, a simple quantum model for wormholes is the coupled SYK model introduced by Maldacena and Qi [14]. The Maldacena-Qi (MQ) model consists of two copies (left/right) of the original SYK model, with additional direct hopping between corresponding modes. Each copy of the SYK model corresponds to a boundary of the AdS space-time. In the low-temperature limit, a traversable wormhole $[29,30]$ between the left and the right boundary is formed. At higher temperatures, there is a first-order transition to a geometry with two disconnected

\footnotetext{
*PengfeiZhang.physics@gmail.com
}

black holes. Dynamical evolution and equilibrium properties of the MQ model have been studied in [34-36]. Later, the model is generalized into the complex fermion version with $\mathrm{U}(1)$ symmetry $[37,38]$ and is found to be related to the large$M$ random spin models [39]. There is also an experimental proposal for realizing the MQ model [40].

In this work, we study the experimental consequence of the eternal traversable wormhole in the bulk from the transport perspective. As in the conventional experimental setup for measuring the tunneling current, we consider attaching leads to each side of the complex MQ model, as shown in Fig. 1. We then apply a voltage at the left lead and measure the current through the right lead. Intuitively, when the MQ

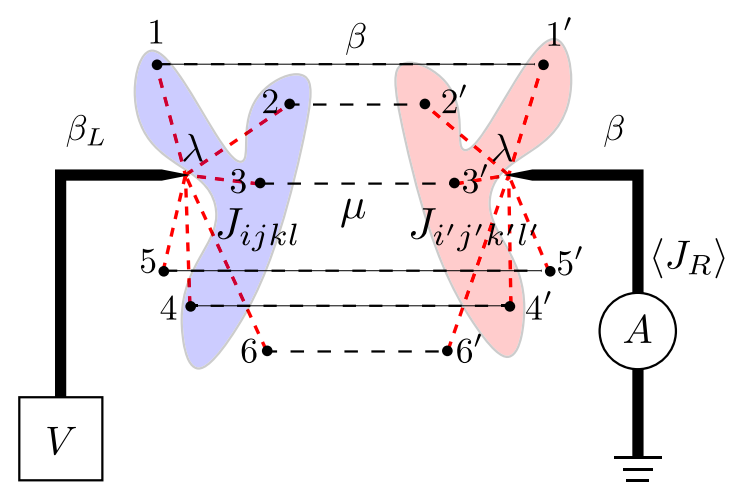

FIG. 1. Schematics of the setup where we couple each side of the MQ model to a lead that allows us to measure the tunneling current. Here the blue/red blob represents specific SYK interaction terms for the $L / R$ copy, which acts nontrivially on four fermion modes. $V$ represents bias voltage added to the left lead and the current on the right lead $J_{R}$ is measured by the ammeter $A$. The inverse temperature is $\beta_{L}$ for the left lead, $\beta$ for the right lead, and $\beta$ for the complex MQ model system. 
model is in the wormhole phase, an electron in the left lead dives into the traversable wormhole and will appear in the right lead, leading to a large tunneling probability when the energy matches the intrinsic modes of the $\mathrm{AdS}_{2}$ space-time. On the other hand, if the MQ model is in the black-hole phase, the correlation between the two sides becomes much weaker, and the tunneling probability becomes small. We show that this intuition is indeed consistent with detailed calculations on either the quantum side or the gravity side, and the tunneling spectroscopy provides an unambiguous signature of the bulk geometry.

\section{MODEL}

We consider coupling each side of the complex MQ model to a different lead described by noninteracting fermions. The Hamiltonian is

$$
\hat{H}=\hat{H}_{\mathrm{MQ}}+\hat{H}_{\text {Lead }}+\sum_{i, p}\left(\lambda_{i} \hat{c}_{L, i}^{\dagger} \hat{\psi}_{L, p}+\lambda_{i} \hat{c}_{R, i}^{\dagger} \hat{\psi}_{R, p}+\text { H.c. }\right),
$$

where $\hat{H}_{\mathrm{MQ}}$ and $\hat{H}_{\text {Lead }}$ read

$$
\begin{aligned}
\hat{H}_{\mathrm{MQ}}= & \sum_{i<j ; k<l} J_{i j, k l}\left(\hat{c}_{L, i}^{\dagger} \hat{c}_{L, j}^{\dagger} \hat{c}_{L, k} \hat{c}_{L, l}+\hat{c}_{R, i}^{\dagger} \hat{c}_{R, j}^{\dagger} \hat{c}_{R, k} \hat{c}_{R, l}\right) \\
& +\mu \sum_{i}\left(\hat{c}_{L, i}^{\dagger} \hat{c}_{R, i}+\hat{c}_{R, i}^{\dagger} \hat{c}_{L, i}\right), \\
\hat{H}_{\text {Lead }}= & \sum_{p} \epsilon_{p}\left(\hat{\psi}_{L, p}^{\dagger} \hat{\psi}_{L, p}+\hat{\psi}_{R, p}^{\dagger} \hat{\psi}_{R, p}\right) .
\end{aligned}
$$

Here $i, j, k, l=1,2, \ldots, N . \hat{c}_{L / R, i}$ is the annihilation operator in the left/right copy of the SYK model, where two copies are coupled by $\mu . \hat{\psi}_{L / R, p}$ describes fermions in the left/right lead with momentum $p$ with a gapless dispersion $\epsilon_{p}$. Note that the number of modes in leads does not scale with $N . J_{i j, k l}$, and $\lambda_{i}$ are independent random Gaussian variables with zero mean and variance:

$$
\overline{\left|J_{i j, k l}\right|^{2}}=\frac{2 J^{2}}{N^{3}}, \quad \overline{\left|\lambda_{i}\right|^{2}}=\frac{\lambda^{2}}{N D} .
$$

Here $D$ is the number of coupled modes in the lead.

We first consider the total system at thermal equilibrium and inverse temperature $\beta$. Since the leads only contain $\mathrm{O}(1)$ modes, to the leading order of $1 / N$, the two-point function of the MQ model is not modified by a finite $\lambda$. Consequently, the system still contains two different phases. In the low-temperature limit, the MQ model is dual to an eternal traversable wormhole, where two copies of the SYK model are connected through a holographic bulk. Here the perfect correlation between two copies plays an important role for obtaining a wormhole solution in the low-temperature limit [14]. At higher temperatures, the system turns into a black-hole phase where the emergent space-times in the gravitational description correspond to nearly disconnected black holes.

We then analyze modes in the leads. Without the coupling to the SYK dots, the left and the right leads are decoupled. When $\lambda$ is turned on, the fermion can tunnel from the left lead to the right lead through the MQ model. Explicitly, we define the retarded Green's function $G_{O}^{\mathcal{R}}(t) \equiv-i \theta(t)\left\langle\left[\hat{O}(t), \hat{O}^{\dagger}(0)\right]\right\rangle$ and focus on the local fermion modes $\hat{\psi}_{\alpha}=\sum_{p} \hat{\psi}_{\alpha, p} / \sqrt{D}$ with $\alpha=L / R$ that couple to the MQ model. In other words, we put the contact point of the lead to the SYK models at $x=0$. Taking into account the self-energy from the MQ model, the Schwinger-Dyson equation of $\psi$ reads

$$
\left(G_{\psi}^{\mathcal{R}}(\omega)^{-1}\right)_{\alpha \gamma}=\left(G_{\psi}^{\mathcal{R}, 0}(\omega)^{-1}\right)_{\alpha \gamma}-\lambda^{2} G_{c}^{\mathcal{R}}(\omega)_{\alpha \gamma} .
$$

Here $G_{c}^{\mathcal{R}}(\omega)_{\alpha \gamma}$ is the retarded Green's function of the MQ model ( $\alpha \gamma$ component). $G_{\psi}^{\mathcal{R}, 0}(\omega)_{\alpha \gamma}=-i \pi \rho_{0} \delta_{\alpha \gamma}$ is the bare Green's function of leads. Here we assume that the density of states of leads can be approximated as a constant $\rho_{0}$. Other real-time Green's functions $G_{\psi}^{\mathcal{P}}$ and $G_{\psi}^{\mathcal{K}}$ are then determined by $G_{\psi}^{\mathcal{A}}(\omega)=G_{\psi}^{\mathcal{R}}(\omega)^{\dagger}$ and at inverse temperature $\beta$ the fluctuation-dissipation theorem gives

$$
G_{\psi}^{\mathcal{K}}(\omega)_{\mathrm{eq}}=\left(G_{\psi}^{\mathcal{R}}(\omega)-G_{\psi}^{\mathcal{A}}(\omega)\right) F_{\beta}(\omega),
$$

where $F_{\beta}(\omega)=1-2 f_{\beta}^{F}(\omega)=\tanh (\beta \omega / 2)$, with $f_{\beta}^{F}(\omega)$ being the Fermi-Dirac distribution function.

\section{TUNNELING SPECTROSCOPY}

Now we consider the nonequilibrium problem of calculating the tunneling current. The charge of the right lead is defined as $\hat{Q}_{R}=\sum_{p} \hat{\psi}_{R, p}^{\dagger} \hat{\psi}_{R, p}$. Consequently, the current flowing from the right SYK model to the right lead reads

$$
\hat{J}_{R}=-i\left[\hat{H}, \hat{Q}_{R}\right]=-i \sum_{i, p}\left(\lambda_{i} \hat{c}_{R, i}^{\dagger} \hat{\psi}_{R, p}-\lambda_{i}^{*} \hat{\psi}_{R, p}^{\dagger} \hat{c}_{R, i}\right) .
$$

When the full system is at thermal equilibrium, the current vanishes. We are interested in the setup shown in Fig. 1, where we tune the voltage $V$ and the inverse temperature $\beta_{L}$ of the left lead while fixing all other parts of the system to $V=0$ and $\beta$. Measuring $\left\langle\hat{J}_{R}\right\rangle$ then detects how many particles from the left lead can tunnel through the MQ model.

The calculation of the tunneling current can be analyzed using the Schwinger-Keldysh formalism. The contours of this path-integral method contain a forward and a backward evolution with fermionic fields $c_{\alpha, i, f / b}$ and $\psi_{\alpha, p, f / b}$. After the Keldysh rotation

$$
c_{\alpha, i, 1 / 2}=c_{\alpha, i, f} \pm c_{\alpha, i, b}, \quad \bar{c}_{\alpha, i, 1 / 2}=\bar{c}_{\alpha, i, f} \mp \bar{c}_{\alpha, i, b},
$$

and similarly for $\psi_{\alpha, p, 1 / 2}$. The Green's function $\mathbf{G}_{O}(\omega)_{\alpha \gamma}^{a b}$ becomes $4 \times 4$ matrices with both $\alpha, \gamma=L / R$ and $a, b=1 / 2$ labels. Explicitly, in 1 / 2 space we have

$$
\mathrm{G}_{\psi}(\omega)_{\alpha \gamma}=\left(\begin{array}{cc}
G_{\psi}^{\mathcal{R}}(\omega)_{\alpha \gamma} & G_{\psi}^{\mathcal{K}}(\omega)_{\alpha \gamma} \\
0 & G_{\psi}^{\mathcal{A}}(\omega)_{\alpha \gamma}
\end{array}\right),
$$

and similarly for $\mathrm{G}_{c}(\omega)_{\alpha \gamma}$. As in Eq. (4), the SchwingerDyson equation for $\mathrm{G}_{\psi}(\omega)$ now reads

$$
\left(\mathbf{G}_{\psi}(\omega)^{-1}\right)_{\alpha \gamma}^{a b}=\left(\mathbf{G}_{\psi}^{0}(\omega)^{-1}\right)_{\alpha \gamma}^{a b}-\lambda^{2}\left(\mathbf{G}_{c}(\omega)\right)_{\alpha \gamma}^{a b} .
$$

Similarly to the equilibrium case, $\mathbf{G}_{c}$ is the equilibrium realtime Green's function of the MQ model, which satisfies the fluctuation-dissipation theorem, Eq. (5), at $\beta$ without additional chemical potential. On the contrary, $\mathrm{G}_{\psi}$ does not satisfy the fluctuation-dissipation theorem since the left lead is at a different temperature and chemical potential. Nevertheless, 
we have relations for the bare Green's functions of leads:

$$
\begin{aligned}
G_{\psi}^{\mathcal{K}, 0}(\omega)_{L L} & =-2 \pi i \rho_{0} F_{\beta_{L}}(\omega-V), \\
G_{\psi}^{\mathcal{K}, 0}(\omega)_{R R} & =-2 \pi i \rho_{0} F_{\beta}(\omega) .
\end{aligned}
$$

Then the tunneling current $\left\langle J_{R}\right\rangle$ can be computed to the leading order under the $1 / N$ expansion. Diagrammatically, we have

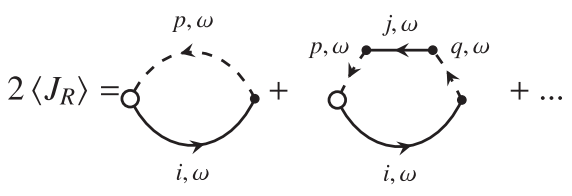

- (Reverse all arrows).

(10)
Here the dashed line represents $\psi$ fields and the solid line represents $c$ fields. The black dots represent the insertion of the identity operator $(I \otimes I)$ in $L / R$ and $1 / 2$ space, while the open circles represent vertex $\lambda^{2}\left(P_{R} \otimes \sigma_{x}\right)$. Here $P_{R}$ is the projector into the $R$ space and $\sigma_{x}$ is in the Pauli matrix in the $1 / 2$ space. Implicitly, all internal labels of modes, momenta, and the frequency $\omega$ should be summed up or integrated over. Summing up all diagrams gives

$$
\left\langle J_{R}\right\rangle=\frac{\lambda^{2}}{2} \int \frac{d \omega}{2 \pi} \operatorname{tr}\left[\mathrm{G}_{c}\left(P_{R} \otimes \sigma_{x}\right) \mathrm{G}_{\psi}-\mathrm{G}_{\psi}\left(P_{R} \otimes \sigma_{x}\right) \mathrm{G}_{c}\right]
$$

Here the trace is taken in both $L / R$ and $1 / 2$ space. Using the explicit formula for $\mathrm{G}_{c}$ and $\mathrm{G}_{\psi}$, we find

$$
\left\langle J_{R}\right\rangle=\int \frac{d \omega}{2 \pi}|T(\omega)|^{2}\left(f_{\beta_{L}}^{F}(\omega-V)-f_{\beta}^{F}(\omega)\right),
$$

with

$$
|T(\omega)|^{2}=\left|\frac{2 \Gamma\left(G_{c}^{\mathcal{R}}\right)_{L R}}{\left(\Gamma\left(G_{c}^{\mathcal{R}}\right)_{L L}-\Gamma\left(G_{c}^{\mathcal{R}}\right)_{L R}-i\right)\left(\Gamma\left(G_{c}^{\mathcal{R}}\right)_{L L}+\Gamma\left(G_{c}^{\mathcal{R}}\right)_{L R}-i\right)}\right|^{2}
$$

Here we have defined $\Gamma=\pi \rho_{0} \lambda^{2} .|T(\omega)|^{2}$ can be understood as the tunneling probability from the left lead to the right lead. Explicitly, the tunneling current is 0 if there is no left-right correlation in the MQ model. In the following sections, we analyze the tunneling current in different phases, focusing on the $\beta_{L}=\beta$ case. One can also derive a similar formula for the energy current with an additional factor of $\omega[8]$.

\section{A. Wormhole phase}

We first consider the system in the zero-temperature limit and focus on small $\mu / J$. Holographically, the MQ model is dual to the eternal traversable wormhole geometry in the global $\mathrm{AdS}_{2}$ space-time with a metric $d s^{2}=\left(-d t_{g}^{2}+\right.$ $\left.d x^{2}\right) / \sin ^{2} x$. The left/right copy of the SYK model lies on the boundary near $x=0$ or $x=\pi$. This indicates that both the diagonal and the off-diagonal components of Green's functions are conformal [14]. At zero temperature, after performing Fourier transform for the conformal Green's functions given in [14], we find

$$
\begin{aligned}
G_{c, \mathrm{WH}}^{\mathcal{R}}(\omega)_{\alpha \alpha} & =-\frac{2 \pi^{5 / 4} \sin (\pi \tilde{\omega}) \sec (2 \pi \tilde{\omega})}{\sqrt{J t^{\prime}} \mathcal{D}_{3 / 4}(\tilde{\omega})}, \\
G_{c, \mathrm{WH}}^{\mathcal{R}}(\omega)_{\alpha \bar{\alpha}} & =-\frac{\mathcal{D}_{1 / 4}(\tilde{\omega})}{\sqrt{2} \pi^{3 / 4} \sqrt{J t^{\prime}}},
\end{aligned}
$$

where $\alpha \neq \bar{\alpha}$. We have defined $\mathcal{D}_{u}(x)=\boldsymbol{\Gamma}(u-x) \boldsymbol{\Gamma}(u+x)$ and $\tilde{\omega} \equiv \omega / t^{\prime}$ for conciseness. Here $\Gamma(z) \equiv \int_{0}^{\infty} d x x^{z-1} e^{-x}$ is the standard gamma function. $t^{\prime}$ is an additional parameter which is proportional to $J^{1 / 3} \mu^{2 / 3}$. It relates the global time $t_{g}$ to the boundary time $t$ as $t_{g}=t^{\prime} t$. The pole of $G_{c}^{\mathcal{R}}(\omega)$ lies at $|\omega|=\omega_{n} \equiv t^{\prime}(1 / 4+n)$ with $n=0,1,2, \ldots$ This tower of the spectrum is fixed by the SL(2) symmetry of the $\mathrm{AdS}_{2}$ space-time.
The full expression for the tunneling probability $|T(\omega)|^{2}$ can then be derived as

$$
|T(\omega)|^{2}=\frac{8}{\frac{4 \pi^{5 / 2} \Gamma^{2}}{J t^{\prime} \mathcal{D}_{3 / 4}(\tilde{\omega})^{2}}+\frac{J t^{\prime} \cos ^{2}(2 \pi \tilde{\omega}) \mathcal{D}_{3 / 4}(\tilde{\omega})^{2}}{\pi^{5 / 2} \Gamma^{2}}-4 \cos (2 \pi \tilde{\omega})+8} .
$$

We plot $|T(\omega)|^{2}$ using the conformal solutions in Fig. 2(a). For extremely small coupling $\Gamma$, the fermion modes can only tunnel through the MQ model when they are on resonance with the MQ model. As a result, we have narrow peaks for the tunneling probability near $\omega_{n}$ with $|T(\omega)|^{2} \approx 1$. This shows that the tunneling current is a direct probe of the eternal traversable wormhole and the underlying SL(2) symmetry. For larger coupling $\Gamma$, the narrow peaks get broadened, and the two peaks at $\pm \omega_{0}$ merge to a single broad peak.

If we further increase $\Gamma$, we find the peaks shift to $\omega_{n}^{\prime} \equiv$ $\pm t^{\prime}(3 / 4+n)$ at small $\omega$. At large $\Gamma$, Eq. (15) asymptotically approaches $\left(2 J t^{\prime} \mathcal{D}_{3 / 4}(\tilde{\omega})^{2}\right) /\left(\pi^{5 / 2} \Gamma^{2}\right)$, which gives rise to the shift of peaks at $\omega_{n}^{\prime}$. Physically, this means that in the large- $\Gamma$ limit, the low-energy tunneling is dominated by the contribution of bulk fields with scaling dimension $3 / 4$, which corresponds to the operator $\hat{\imath}_{\alpha, i}=\sum_{j ; k<l} J_{i j, k l} \hat{c}_{\alpha, j}^{\dagger} \hat{c}_{\alpha, k} \hat{c}_{\alpha, l}$. Similar effects have been studied in the SYK chain model [41] and give rise to the low-high voltage duality for the tunneling spectroscopy of the single SYK model [7].

We then consider the finite temperature and the finite $\mu / J$ corrections within the wormhole phase. In this case, we numerically solve the retarded Green's functions for the MQ model $[34,35]$ and then compute the tunneling probability $|T(\omega)|^{2}$. As found in $[34,35]$, at finite temperature, the realtime Green's functions decay and there is a finite lifetime for quasiparticle modes. Moreover, the weight of modes decays quickly when $n$ increases. Consequently, as shown in Fig. 2(b), the tunneling probability, although still showing a 
(a)

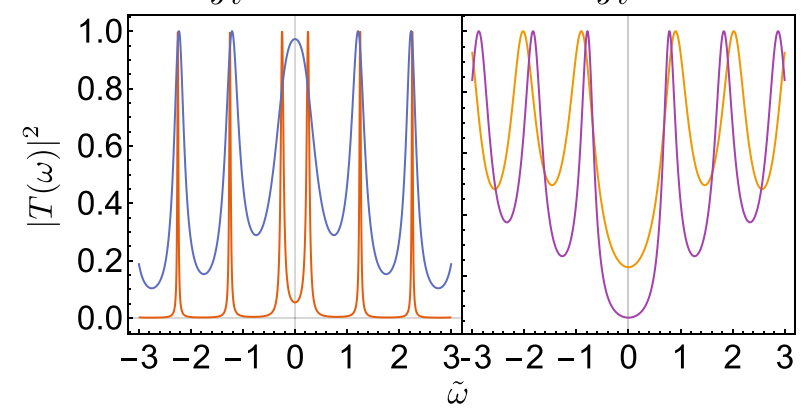

(b)
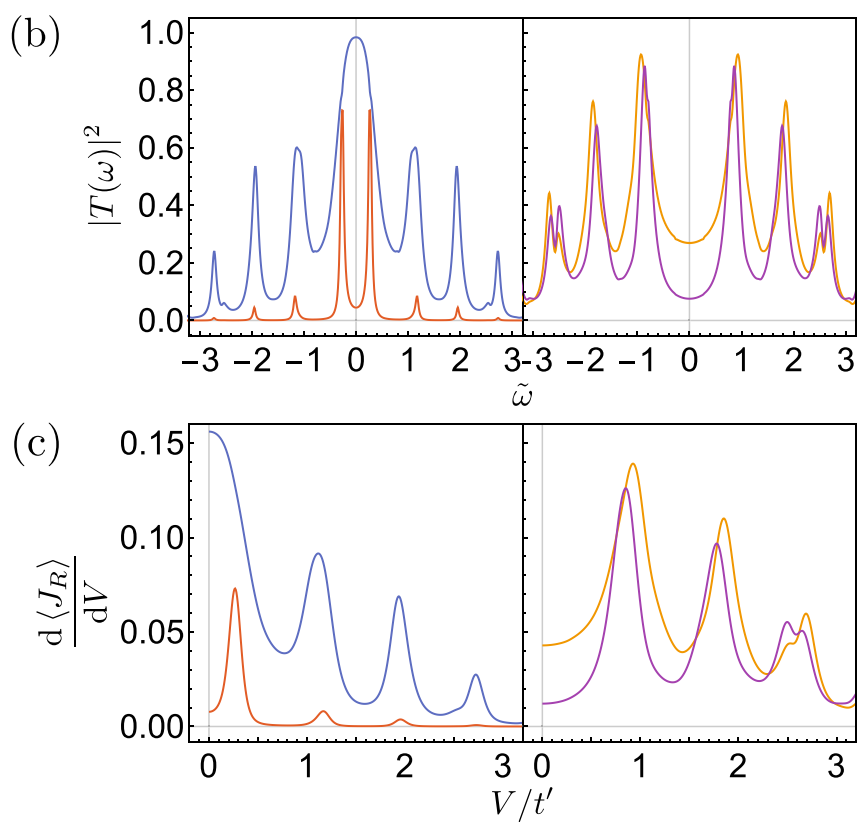

FIG. 2. (a) The tunneling probability obtained from conformal solutions, Eq. (15). (b) The tunneling probability obtained by directly using the solutions of the Schwinger-Dyson equation in Eq. (13). The Green's functions are self-consistently calculated in $\beta J=120$ and $\mu / J=0.025$, which results in $t^{\prime} / J=0.3$. (c) The differential conductance defined by taking the derivative of Eq. (12). We also use the same Green's functions as in (b). In (a)-(c), the left panel exhibits peaks at $\omega_{n} \equiv t^{\prime}(n+1 / 4)$ and the right panel exhibits peaks at $\omega_{n}^{\prime} \equiv t^{\prime}(n+3 / 4)$.

few peaks, becomes smaller than 1 when $n$ becomes large. We also plot the differential conductance $d\left\langle J_{R}\right\rangle / d V$ in Fig. 2(c). As an essential physical quantity in condensed matter experiments, we find that differential conductance measurement at finite temperature also shows multiple peaks at $\omega_{n}$ or $\omega_{n}^{\prime}$, providing the prominently nontrivial predictions for the experiments.

\section{B. Black-hole phase}

If we increase the temperature $T \gtrsim \mu$, there is a first-order transition in the MQ model after which the system gets into the black-hole phase where each side lives on the boundary of a separate Rindler space-time with $d s^{2}=-r(r-2) d t_{R}^{2}+$
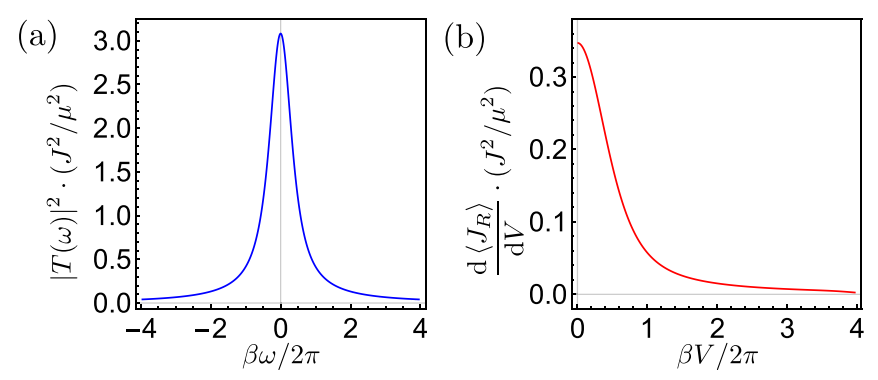

FIG. 3. (a) The tunneling probability and (b) the differential conductance in the black-hole phase for $\Gamma / J=0.3$ and $\beta J=5$.

$d r^{2} / r(r-2)$. This is an analogy of the Hawking-Page transition in higher dimensions. In the black-hole phase, from the gravity perspective, the Rindler space-times of the black holes emulated by left/right SYK models are disconnected. Adding the quantum correlation propotional to $\mu$, we can approximate

$$
G_{c, \mathrm{BH}}^{\mathcal{R}}(\omega)_{\alpha \alpha}=-i \frac{\sqrt{\beta / J}}{\sqrt{2} \pi^{1 / 4}} \frac{\Gamma\left(\frac{1}{4}-i \frac{\omega \beta}{2 \pi}\right)}{\Gamma\left(\frac{3}{4}-i \frac{\omega \beta}{2 \pi}\right)},
$$

and $G_{c, \mathrm{BH}}^{\mathcal{R}}(\omega)_{\alpha \bar{\alpha}}=\mu\left(G_{c, \mathrm{BH}}^{\mathcal{R}}(\omega)_{\alpha \alpha}\right)^{2}$. We have kept terms up to order $\mu$ and assume $\beta J \gg 1$. We can then expand $|T(\omega)|^{2}$ to the $O\left(\mu^{2}\right)$ order and obtain

$$
|T(\omega)|^{2}=\frac{4 \mu^{2} / \Gamma^{2}}{\left|1+\sqrt{\frac{2 J \pi^{1 / 2}}{\beta \Gamma^{2}}} \frac{\Gamma\left(\frac{3}{4}-i \frac{\omega \beta}{2 \pi}\right)}{\Gamma\left(\frac{1}{4}-i \frac{\omega \beta}{2 \pi}\right)}\right|^{4}} .
$$

Differently from the wormhole phase, the spectral function is now a single peak near $\omega \sim 0$. We expect similar behavior for the tunneling probability, as verified in Fig. 3(a). We also plot differential conductance $d\left\langle J_{R}\right\rangle / d V$ in Fig. 3(b), which, in contrast with the wormhole phase, shows no oscillating behavior. Plus, according to Eq. (17), the magnitude of the tunneling probability is proportional to $(\mu / J)^{2}$ in the small$\mu / J$ limit and is much smaller than the wormhole phase result in the order of magnitude 1.

\section{HOLOGRAPHIC PICTURE}

Now we analyze the problem from a bulk perspective. To get a simple holographic picture, we now choose a specific dispersion $\epsilon_{p}$. We assume that each lead can be described as a half-infinite line with both massless left-moving and massless right-moving Dirac fields living on a flat space-time; we show that a gravity calculation can reproduce previous results.

We first give a brief introduction of the holographic dictionary [1]. The holographic duality states that there is an equivalence between a strongly correlated quantum manybody system and a semiclassical system with gravity. The best understood example is the duality between an AdS gravity theory with space-time dimension $d+1$ and a conformal field theory with space-time dimension $d$, where the isometry group of the AdS space-time matches the conformal group of the quantum theory. Mathematically, the holographic duality says

$$
\int D \phi e^{i S_{\mathrm{QFT}}[\phi]+\int J O}=\left.e^{i S_{\text {bulk }}[\Phi, g]}\right|_{\Phi \rightarrow J},
$$


(a)

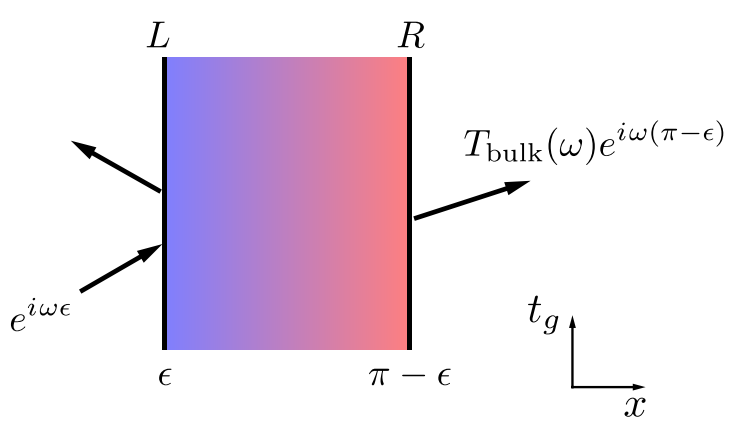

(b)

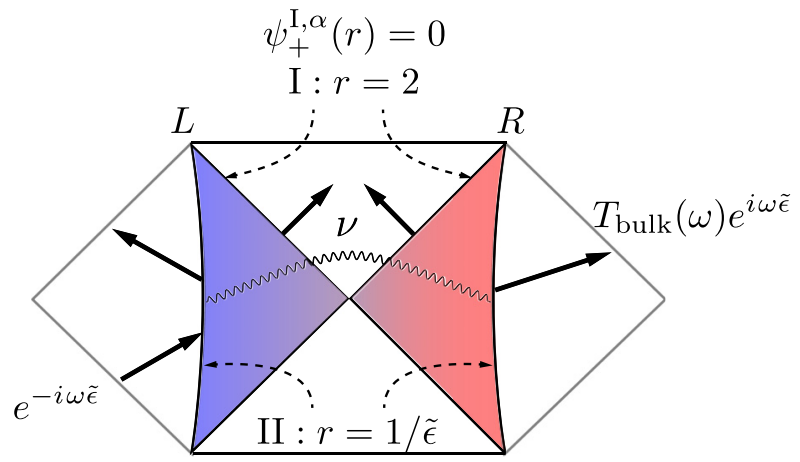

FIG. 4. The gravity picture for the tunneling probability in the (a) wormhole geometry and (b) black-hole geometry. Here the colored region in (a) corresponds to the $\mathrm{AdS}_{2}$ space-time, and the colored region in (b) corresponds to two copies of the Rindler spacetime. In both cases, we consider an ingoing Dirac fermion from the left lead and determine the tunneling amplitude $T_{\text {bulk }}(\omega)$ by solving the Dirac equation. In (b), the wiggle line corresponds to the coupling $v$ introduced in Eq. (37), and we impose the in-falling boundary condition $\psi_{+}^{\mathrm{I}, \alpha}(r)=0$ at horizon $r=2$.

which is known as the GKPW formula. Here on the left-hand side, we have a path integral for quantum fields $\phi$, with external source coupled $J$ to the field $O$. On the right-hand side, we take the saddle-point solution of a gravity theory, where we require that the boundary value of bulk fields should be equal to $J$, which is specified in more detail below. This formula enables us to compute the correlation function of a quantum system by solving classical equations.

To illustrate how a holographic calculation works, we consider reproducing the Green's function, Eq. (16), of the single SYK model from a bulk calculation. The calculation here is standard but may benefit general readers. The single SYK model in the low-energy limit is a conformal field theory in $0+1$ dimension, which, as mentioned in the last section, is dual to the $\mathrm{AdS}_{2}$ Rindler geometry with metric $d s^{2}=-r(r-$ 2) $d t_{R}^{2}+d r^{2} / r(r-2)$. This geometry can be illustrated as the left side of Fig. 4(b). This means that one should imagine the bulk action contains some gravity part, with a solution of the corresponding metric. We then just need to solve the equation of motion for bulk fields in this geometry. Since Eq. (16) is the Green's function for fermionic operators, it is natural to consider the Dirac equation in the bulk:

$$
S_{\text {bulk }}=\int \sqrt{-g} d r d t_{R}(i \bar{\Psi} \not D \Psi-m \bar{\Psi} \Psi) .
$$

Here we have included the mass $m$, which, as we see, is related to the scaling dimension of the operator $\Delta$ in the quantum the- ory as $m=1 / 2-\Delta$. The Dirac equation in Rindler geometry can be solved exactly and the full expression can be found in the Appendix. Here we only focus on certain limits.

A general solution for the Dirac equation contains two independent coefficients $C_{1}$ and $C_{2}$, whose relation should be determined from the boundary conditions. The space-time contains two important limits: I, the horizon at $r \rightarrow 2$; and II, the space-time boundary at $r \rightarrow \infty$. We first consider the solution near the horizon. Expanding the general solution, (A6), around $r=2$, one finds

$$
\begin{aligned}
\psi_{-}^{\mathrm{I}}(r)= & \left(C_{1}+C_{2}\right) \frac{2^{-\frac{1}{4}+\frac{i \omega}{2}} \pi(r-2)^{-\frac{1}{4}-\frac{i \omega}{2}} \csc \left(\pi\left(\frac{1}{2}+i \omega\right)\right)}{\boldsymbol{\Gamma}\left(\frac{1}{2}-i \omega\right) \boldsymbol{\Gamma}\left(i \omega+\frac{1}{2}\right)}, \\
\psi_{+}^{\mathrm{I}}(r)= & \frac{2^{-\frac{1}{4}-\frac{i \omega}{2}} \pi(r-2)^{-\frac{1}{4}+\frac{i \omega}{2}} \csc \left(\pi\left(\frac{1}{2}-i \omega\right)\right)}{\boldsymbol{\Gamma}\left(i \omega+\frac{1}{2}\right)^{2}} \\
& \times\left(C_{1} \frac{\boldsymbol{\Gamma}\left(i \omega+\frac{1}{2}+m\right)}{\boldsymbol{\Gamma}\left(\frac{1}{2}+m-i \omega\right)}-C_{2} \frac{\boldsymbol{\Gamma}\left(i \omega+\frac{1}{2}-m\right)}{\boldsymbol{\Gamma}\left(\frac{1}{2}-m-i \omega\right)}\right) .
\end{aligned}
$$

Here we use the label I to represent the Dirac field near the horizon $r=2$, as shown in Fig. 4(b). From the factor of $(r-2)^{ \pm i \omega / 2}$, we find that $\psi_{-}^{\mathrm{I}}$ represents a wave moving towards $r=2$, which is called an in-falling solution, and $\psi_{+}^{\mathrm{I}}$ represents a wave moving outwards, which is called an outgoing solution. Physically, one might expect that a physical object should only contain in-falling components, which means that we should impose the boundary condition that $\psi_{+}^{\mathrm{I}}=0$ when computing the retarded Green's function [1]. This determines the relation between $C_{1}$ and $C_{2}$ :

$$
\frac{C_{1}}{C_{2}}=\frac{\boldsymbol{\Gamma}\left(\frac{1}{2}+m-i \omega\right) \boldsymbol{\Gamma}\left(i \omega+\frac{1}{2}-m\right)}{\boldsymbol{\Gamma}\left(\frac{1}{2}-m-i \omega\right) \boldsymbol{\Gamma}\left(i \omega+\frac{1}{2}+m\right)} .
$$

We then study the asymptotic behavior of the solution at the AdS boundary at $r \rightarrow \infty$. The result reads

$$
\begin{aligned}
\left(\begin{array}{c}
\psi_{\overline{\mathrm{II}}}^{\mathrm{II}} \\
\psi_{+}^{\mathrm{II}}
\end{array}\right)= & C_{2} \frac{r^{-\frac{1}{2}+m} \boldsymbol{\Gamma}(1-m) \boldsymbol{\Gamma}\left(\frac{1}{2}-m+i \omega\right)}{2^{m} \boldsymbol{\Gamma}(1-2 m) \boldsymbol{\Gamma}\left(\frac{1}{2}+i \omega\right)}\left(\begin{array}{c}
1 \\
-1
\end{array}\right) \\
& +C_{1} \frac{r^{-\frac{1}{2}-m} \boldsymbol{\Gamma}(1+m) \boldsymbol{\Gamma}\left(\frac{1}{2}+m+i \omega\right)}{2^{-m} \boldsymbol{\Gamma}(1+2 m) \boldsymbol{\Gamma}\left(\frac{1}{2}+i \omega\right)}\left(\begin{array}{l}
1 \\
1
\end{array}\right) .
\end{aligned}
$$

Likewise, we use the label II for the result expanded near $r=\infty$. Now we should specify what is the boundary value of the bulk fields. Near $r \rightarrow \infty$, we see that there are two independent solutions proportional to $r^{-1 / 2+m}(1,-1)$ and $r^{-1 / 2-m}(1,1)$. The boundary value of the bulk field is defined as the coefficient of the solution $r^{-1 / 2-m}(1,1)$. This relates the source $J(\omega)$ and $C_{1}$,

$$
J(\omega)=C_{1} \frac{\boldsymbol{\Gamma}(1+m) \boldsymbol{\Gamma}\left(\frac{1}{2}+m+i \omega\right)}{2^{-m} \boldsymbol{\Gamma}(1+2 m) \boldsymbol{\Gamma}\left(\frac{1}{2}+i \omega\right)},
$$

which fixes the solution, Eq. (A6), in the bulk together with Eq. (21).

One can then determine the right-hand side of Eq. (18) by using the solution, with additional boundary counter-terms for the holographic renormalization [1]. The result shows that there is a shortcut to this calculation, where the coefficient of the other solution near $r \rightarrow \infty$ is just proportional to the ex- 
pectation of the boundary operator $\langle O(\omega)\rangle_{J}$ with finite source term $J$ :

$$
\langle O(\omega)\rangle_{J} \propto C_{2} \frac{\boldsymbol{\Gamma}(1-m) \boldsymbol{\Gamma}\left(\frac{1}{2}-m+i \omega\right)}{2^{m} \boldsymbol{\Gamma}(1-2 m) \boldsymbol{\Gamma}\left(\frac{1}{2}+i \omega\right)} .
$$

The Green's function is then determined by

$$
G_{O O}^{R}(\omega)=\frac{\langle O(\omega)\rangle_{J}}{J(\omega)} \propto \frac{4^{m} \boldsymbol{\Gamma}\left(m+\frac{1}{2}\right) \boldsymbol{\Gamma}\left(-m-i \omega+\frac{1}{2}\right)}{\boldsymbol{\Gamma}\left(\frac{1}{2}-m\right) \boldsymbol{\Gamma}\left(m-i \omega+\frac{1}{2}\right)} .
$$

This exactly takes the form of the conformal Green's function in $0+1$ dimension with a scaling dimension $\Delta=1 / 2-m$, which matches Eq. (16) for $m=1 / 4$, if we also make the substitution $\omega \rightarrow \omega \beta / 2 \pi$ due to the relation between the boundary and the global Rindler time $t=\beta t_{R} / 2 \pi$.

Similarly, one can consider the wormhole geometry, where there are two boundaries. The solution of Dirac equations can also be derived analytically. Its asymptotic form near each boundary also contains two terms with different powers of coordinate as in Eq. (22), whose coefficients determine the value of the source term and the operator expectation. Similar calculations on a global AdS can reproduce Eq. (14).

\section{A. Wormhole geometry}

Having introduced the basic idea of holographic calculations, we are now ready to explain the calculation of the tunneling probability. We first focus on the wormhole phase where the correlation between the left and the right system is from a nontrivial bulk geometry. The MQ model in the wormhole phase is equivalent to a fermionic field in the global $\mathrm{AdS}_{2}$ space-time with mass $m=1 / 2-\Delta$. Recall that we assume the leads are described by massless Dirac fermions in flat space-time, and we consider the following action of bulk Dirac fermions on the geometry shown in Fig. 4(a),

$$
S_{\text {bulk }}^{\mathrm{WH}}=\int \sqrt{-g} d x d t_{g}(i \bar{\Psi} \not D \Psi-m(x) \bar{\Psi} \Psi),
$$

where $\not D$ is the covariant derivative of spinors on curved space-time. The metric $d s^{2}=\left(-d t_{g}^{2}+d x^{2}\right) / \zeta^{2}(x)$ and the mass $m(x)$ depend on the spatial coordinate as

$$
(\zeta(x), m(x))= \begin{cases}(\epsilon, 0), & x \in(-\infty, \epsilon) \cup(\pi-\epsilon, \infty), \\ (\sin x, 1 / 4), & x \in(\epsilon, \pi-\epsilon) .\end{cases}
$$

Here $\epsilon$ is a cutoff where we glue different geometries and it should be related to $\Gamma$. We also assume that $\epsilon \ll 1$ and neglect higher orders of $\epsilon$. As we see, this corresponds to a large coupling $\lambda$ in the original model. Note that we have assumed a solid background of $\mathrm{AdS}_{2}$ geometry for the MQ model, which is a direct consequence of having a small number of probe fields. When the external field contains $O(N)$ degrees of freedom, there will be back-reaction, which changes the $\mathrm{AdS}_{2}$ geometry.

The tunneling probability $|T(\omega)|^{2}$ corresponds to a scattering problem in the gravity theory. Writing the Dirac field as $\Psi=\left(\psi_{-}, \psi_{+}\right)$, in the $x<\epsilon$ region, we consider an ingoing right-moving fermion $\psi_{+}(x)=e^{i \omega x}$ with momentum $p=$ $\omega$ and a reflected left-moving fermion $\psi_{-}(x)=R(\omega) e^{-i \omega x}$.
In the $x>\pi-\epsilon$ region, only a right-moving component $\psi_{+}(x)=T_{\text {bulk }}(\omega) e^{i \omega x}$ exists.

To determine $T_{\text {bulk }}(\omega)$, we need to match the boundary condition between the global AdS space-time and the flat space-time by requiring the Dirac field is smooth. The complete solution of the Dirac equation in this coordinate is given in the Appendix. In the limit of small $\epsilon$, we only need the asymptotic behavior of $\Psi$ near $x=0$ and $x=\pi$. Near $x=0$, we have the expansion for the spinor fields

$$
\begin{aligned}
& \psi_{1}^{L}(x)=2^{-1 / 4} \tilde{C}_{1} x^{1 / 4}, \\
& \psi_{2}^{L}(x)=2^{1 / 4} \tilde{C}_{2} x^{-1 / 4},
\end{aligned}
$$

while expanding around $x=\pi$, we find

$$
\begin{aligned}
& \psi_{1}^{R}(x)=\frac{2^{3 / 4} \pi}{(\pi-x)^{1 / 4}}\left(\frac{\tilde{C_{1}} \boldsymbol{\Gamma}\left(\frac{3}{4}\right)}{\boldsymbol{\Gamma}\left(\frac{1}{4}\right) \mathcal{D}_{3 / 4}(\omega)}+\frac{i \tilde{C}_{2} \omega}{\mathcal{D}_{1}(\omega)}\right), \\
& \psi_{2}^{R}(x)=2^{1 / 4} \pi(\pi-x)^{1 / 4}\left(\frac{\tilde{C_{2}} \boldsymbol{\Gamma}\left(\frac{1}{4}\right)}{\boldsymbol{\Gamma}\left(\frac{3}{4}\right) \mathcal{D}_{1 / 4}(\omega)}+\frac{i \tilde{C}_{1} \omega}{\mathcal{D}_{1}(\omega)}\right) .
\end{aligned}
$$

Here $L / R$ represents that the general solutions with constants $\tilde{C}_{1}$ and $\tilde{C}_{2}$ are expanded around $x=0$ or $x=\pi$. As we define in the Appendix, the linear combinations of these spinor fields give rise to the left-moving or right-moving component at each boundary. The continuous condition of the left-moving component at the right boundary $x=\pi-\epsilon$ leads to the constraint $\psi_{-}^{R}(\pi-\epsilon)=\left(\psi_{1}^{R}(\pi-\epsilon)-\psi_{2}^{R}(\pi-\right.$ $\epsilon)) /(2 i)=0$, which finally can be simplified as

$$
\frac{\tilde{C}_{1}}{\tilde{C}_{2}}=\frac{\sqrt{2}\left(\mathcal{D}_{3 / 4}(0) \cos (2 \pi \omega) \mathcal{D}_{1 / 4}(\omega)-2 i \pi^{2} \sqrt{\epsilon} \sin (\pi \omega)\right)}{\sqrt{\epsilon} \mathcal{D}_{1 / 4}(0) \cos (2 \pi \omega) \mathcal{D}_{3 / 4}(\omega)-4 i \pi^{2} \sin (\pi \omega)} .
$$

Moreover, the continuous conditions of the right-moving component at the left or right boundary give that

$$
\begin{aligned}
e^{i \omega \epsilon} & =\left(\psi_{1}^{L}(\epsilon)+\psi_{2}^{L}(\epsilon)\right) / 2, \\
T_{\text {bulk }}(\omega) e^{i \omega(\pi-\epsilon)} & =\left(\psi_{1}^{R}(\pi-\epsilon)+\psi_{2}^{R}(\pi-\epsilon)\right) / 2 .
\end{aligned}
$$

These two equations determine the tunneling probability $\left|T_{\text {bulk }}(\omega)\right|^{2}$ as

$$
\begin{aligned}
\left|T_{\text {bulk }}(\omega)\right|^{2} & =\left|e^{i \omega(2 \epsilon-\pi)} \frac{\psi_{1}^{R}(\pi-\epsilon)+\psi_{2}^{R}(\pi-\epsilon)}{\psi_{1}^{L}(\epsilon)+\psi_{2}^{L}(\epsilon)}\right|^{2} \\
& =\frac{8}{\frac{4 \mathcal{D}_{3 / 4}(0)^{2}}{\epsilon \mathcal{D}_{3 / 4}(\omega)^{2}}+\frac{\epsilon \cos ^{2}(2 \pi \omega) \mathcal{D}_{3 / 4}(\omega)^{2}}{\mathcal{D}_{3 / 4}(0)^{2}}-4 \cos (2 \pi \omega)+8}
\end{aligned}
$$

Finally, we find that $\left|T_{\text {bulk }}(\omega)\right|^{2}$ becomes exactly the same as Eq. (15) once we identify $\epsilon=J t^{\prime} \mathcal{D}_{3 / 4}(0)^{2} / \pi^{5 / 2} \Gamma^{2}$ and recall that in the bulk calculation we are measuring energy with respect to $t_{g}$, which differs from the boundary energy $t$ by a factor of $t^{\prime}$, i.e., $t_{g}=t^{\prime} t$, and the $\omega$ here corresponds to $\tilde{\omega}$ in Eq. (15).

\section{B. Black-hole geometry}

We then consider the bulk calculation in the black-hole phase. Geometrically, the left and the right system are decoupled and each side of the MQ model can be replaced by a massive Dirac fermion in the Rindler space-time. The bulk 
action then reads

$$
S_{\text {bulk }}^{\mathrm{BH}}=\sum_{\alpha=L / R} \int \sqrt{-g} d r d t_{R}\left(i \bar{\Psi}_{\alpha} / D \Psi_{\alpha}-m(r) \bar{\Psi}_{\alpha} \Psi_{\alpha}\right),
$$

with $d s^{2}=-d t_{R}^{2} / \zeta^{2}(r)+\zeta^{2}(r) d r^{2}$ and $m(r)$ being

$$
(\zeta(r), m(r))= \begin{cases}(\tilde{\epsilon}, 0), & r \in(1 / \tilde{\epsilon}, \infty) ; \\ (1 / \sqrt{r(r-2)}, 1 / 4), & r \in(2,1 / \tilde{\epsilon}) .\end{cases}
$$

Here boundary I, $r=2$, is the location of the horizon, and the Rindler space-time is connected to the flat space-time at boundary II, $r=1 / \tilde{\epsilon}$, where $\tilde{\epsilon}$ is not necessarily the same as $\epsilon$. The boundary time $t$ is related to $t_{R}$ as $t=\beta t_{R} / 2 \pi$. Furthermore, to distinguish the left $(L)$ and right $(R)$ systems, we introduce additional labels $\alpha=L / R$ on spinor fields and constants. Equation (20) and Eq. (22) now read

$$
\begin{aligned}
\psi_{-}^{\mathrm{I}, \alpha}(r)= & \left(C_{1}^{\alpha}+C_{2}^{\alpha}\right) \frac{2^{-\frac{1}{4}+\frac{i \omega}{2}} \pi(r-2)^{-\frac{1}{4}-\frac{i \omega}{2}} \csc \left(\pi\left(\frac{1}{2}+i \omega\right)\right)}{\boldsymbol{\Gamma}\left(\frac{1}{2}-i \omega\right) \boldsymbol{\Gamma}\left(i \omega+\frac{1}{2}\right)} \\
\psi_{+}^{\mathrm{I}, \alpha}(r)= & \frac{2^{-\frac{1}{4}-\frac{i \omega}{2}} \pi(r-2)^{-\frac{1}{4}+\frac{i \omega}{2}} \csc \left(\pi\left(\frac{1}{2}-i \omega\right)\right)}{\boldsymbol{\Gamma}\left(i \omega+\frac{1}{2}\right)^{2}} \\
& \times\left(C_{1}^{\alpha} \frac{\boldsymbol{\Gamma}\left(i \omega+\frac{1}{2}+m\right)}{\boldsymbol{\Gamma}\left(\frac{1}{2}+m-i \omega\right)}-C_{2}^{\alpha} \frac{\boldsymbol{\Gamma}\left(i \omega+\frac{1}{2}-m\right)}{\boldsymbol{\Gamma}\left(\frac{1}{2}-m-i \omega\right)}\right) \\
\left(\begin{array}{l}
\psi_{-}^{\mathrm{II}}, \alpha \\
\psi_{+}^{\mathrm{II}}, \alpha
\end{array}\right)= & C_{2}^{\alpha} \frac{r^{-\frac{1}{2}+m} \boldsymbol{\Gamma}(1-m) \boldsymbol{\Gamma}\left(\frac{1}{2}-m+i \omega\right)}{2^{m} \boldsymbol{\Gamma}(1-2 m) \boldsymbol{\Gamma}\left(\frac{1}{2}+i \omega\right)}\left(\begin{array}{c}
1 \\
-1
\end{array}\right) \\
& +C_{1}^{\alpha} \frac{r^{-\frac{1}{2}-m} \boldsymbol{\Gamma}(1+m) \boldsymbol{\Gamma}\left(\frac{1}{2}+m+i \omega\right)}{2^{-m} \boldsymbol{\Gamma}(1+2 m) \boldsymbol{\Gamma}\left(\frac{1}{2}+i \omega\right)}\left(\begin{array}{c}
1 \\
1
\end{array}\right) .
\end{aligned}
$$

Up to now, the left and right copies are still decoupled. To have a nonvanishing contribution, we further need to add the coupling between the boundary II in different copies:

$$
\Delta S_{\text {bulk }}^{\mathrm{BH}} \sim v \int \sqrt{-\gamma} d t_{R}\left(\overline{\Psi_{L}^{B}} \Psi_{R}^{B}+\text { H.c. }\right)
$$

Here $\Psi_{\alpha}^{B}$ is the corresponding boundary operator at side $\alpha$ and $\gamma$ is the reduced metric on the boundary. The coupling constant $v$ should be proportional to $\mu$. This geometry is illustrated in Fig. 4(b).

The tunneling probability can now be determined perturbatively. When $v=0$, by again imposing the in-falling boundary condition near the horizon, we can compute an ingoing Dirac fermion in the left lead scattered by the left black hole. The metric in flat space, defined in Eq. (34), reveals that the ingoing Dirac fermion is a mode $e^{-i \omega \tilde{\epsilon}^{2} r}$ in the left lead which moves towards $r=2$. Note that the sign of the ingoing mode is determined by the convention of coordinates. Here the ingoing mode moves to the direction that $r$ decreases, in contrast to the wormhole phase where the ingoing mode moves to the direction where $x$ increases, as denoted in Figs. 4(a) and 4(b). The matching between the mode and Eq. (22) at $r=1 / \tilde{\epsilon}$ gives

$$
e^{-i \omega \tilde{\epsilon}}=\psi_{-}^{\mathrm{II}, L}(1 / \tilde{\epsilon}) .
$$

Here we have added the $L / R$ indices for the bulk fields to distinguish two Rindler space-times. Together with the result of in-falling boundary conditions,

$$
\frac{C_{1}^{\alpha}}{C_{2}^{\alpha}}=\frac{\boldsymbol{\Gamma}\left(\frac{1}{2}+m-i \omega\right) \boldsymbol{\Gamma}\left(i \omega+\frac{1}{2}-m\right)}{\boldsymbol{\Gamma}\left(\frac{1}{2}-m-i \omega\right) \boldsymbol{\Gamma}\left(i \omega+\frac{1}{2}+m\right)},
$$

Eq. (38) determines the wave function $\left(\psi_{-}^{L}(r), \psi_{+}^{L}(r)\right)$ on the left copy.

We then take the coupling, Eq. (37), into account perturbatively. The coupling term can be understood as a source term on the right boundary, whose strength is proportional to $v$ times the expectation of the left system boundary operator [1], i.e., the coefficient in Eq. (36) that is proportional to $r^{-1 / 2+m}$. This gives

$$
\delta J^{R}=v \frac{C_{2}^{L} \boldsymbol{\Gamma}\left(\frac{3}{4}\right) \Gamma\left(i \omega+\frac{1}{4}\right)}{2^{1 / 4} \sqrt{\pi} \Gamma\left(i \omega+\frac{1}{2}\right)} .
$$

With this additional contribution, we can determine two continuous conditions at the right copy to the leading order of $v$. First, the absence of the ingoing wave at boundary II of the right copy ensures that $(1 / \tilde{\epsilon})^{-3 / 4} \delta J^{R}+\psi_{-}^{\mathrm{II}, R}(1 / \tilde{\epsilon})=0$. This can be written explicitly:

$$
\frac{2^{3 / 2} \boldsymbol{\Gamma}\left(\frac{5}{4}\right) \boldsymbol{\Gamma}\left(i \omega+\frac{3}{4}\right)}{\boldsymbol{\Gamma}\left(\frac{3}{4}\right) \boldsymbol{\Gamma}\left(i \omega+\frac{1}{4}\right)} C_{1}^{R}+(\tilde{\epsilon})^{-1 / 2} C_{2}^{R}-v C_{2}^{L}=0 .
$$

Second, the outgoing wave can be similarly calculated as $T_{\text {bulk }}(\omega) e^{i \omega \tilde{\epsilon}}=(1 / \tilde{\epsilon})^{-3 / 4} \delta J^{R}+\psi_{+}^{\mathrm{II}, R}(1 / \tilde{\epsilon})$. Finally, we obtain the tunneling probability:

$$
\begin{aligned}
\left|T_{\text {bulk }}\right|^{2} & =\left|\frac{(1 / \tilde{\epsilon})^{-3 / 4} \delta J^{R}+\psi_{+}^{\mathrm{II}, R}(1 / \tilde{\epsilon})}{\psi_{-}^{\mathrm{II}, L}(1 / \tilde{\epsilon})}\right|^{2} \\
& =\frac{4 v^{2} \tilde{\epsilon}}{\left|1+\frac{\sqrt{\tilde{\epsilon}} \boldsymbol{\Gamma}\left(\frac{1}{4}\right) \Gamma\left(\frac{3}{4}-i \omega\right)}{\sqrt{2} \boldsymbol{\Gamma}\left(\frac{3}{4}\right) \Gamma\left(\frac{1}{4}-i \omega\right)}\right|^{4}} .
\end{aligned}
$$

By identifying

$$
v=\mu /(\Gamma \sqrt{\tilde{\epsilon}}), \quad \tilde{\epsilon}=4 \sqrt{\pi} J \Gamma\left(\frac{3}{4}\right)^{2} /\left(\beta \Gamma^{2} \Gamma\left(\frac{1}{4}\right)^{2}\right)
$$

and again making the substitution $\omega \rightarrow \omega \beta / 2 \pi$, we find that $\left|T_{\text {bulk }}(\omega)\right|^{2}$ matches the result [Eq. (17)] on the quantum side exactly.

\section{CONCLUSION}

We consider the tunneling spectroscopy for the MQ model by coupling each side to a different lead. In the lowtemperature wormhole phase and for small coupling to leads, both the tunneling probability $|T(\omega)|^{2}$ and the differential conductance $d J_{R}(V) / d V$ show peaks at $\left|\omega_{n}\right|=t^{\prime}(1 / 4+n)$, which is fixed by the SL(2) symmetry. In the high-temperature black-hole phase, there is only a single peak near $\omega=0$. We further give a holographic picture in both phases and find an exact match for the calculation between the gravity side and the quantum side.

There are several extensions of the current work. One can consider adding chemical potential to the complex version of the MQ model, which should be dual to adding gauge fields in the bulk. It is also interesting to consider a large number 
of modes in the leads, and then there will be nontrivial backreaction for the $\mathrm{AdS}_{2}$ background and the problem should be solved self-consistently. We defer these to further studies.

\section{ACKNOWLEDGMENTS}

We thank Yingfei $\mathrm{Gu}$ for helpful discussions. P.Z. also thanks Yiming Chen and Xiao-Liang Qi for valuable discussions when collaborating on previous works [42,43]. P.Z. acknowledges support from the Walter Burke Institute for Theoretical Physics at Caltech.

\section{APPENDIX: THE SOLUTION OF THE DIRAC EQUATION}

In this Appendix, we give the general solutions of the Dirac equation in both the wormhole geometry and the black-hole geometry. We first consider the global $\mathrm{AdS}_{2}$ space-time for the wormhole geometry with metric $d s^{2}=\left(-d t_{g}^{2}+d x^{2}\right) / \sin ^{2} x$. In terms of left-moving and right-moving components $\Psi=$ $\sqrt{\sin x}\left(\psi_{-}, \psi_{+}\right)$, the Dirac equation reads

$$
\begin{aligned}
& i\left(\partial_{t_{g}}+\partial_{x}\right) \psi_{+}\left(t_{g}, x\right)=-m \psi_{-}\left(t_{g}, x\right) \csc (x), \\
& i\left(\partial_{t_{g}}-\partial_{x}\right) \psi_{-}\left(t_{g}, x\right)=-m \psi_{+}\left(t_{g}, x\right) \csc (x) .
\end{aligned}
$$

Specifically, left-moving is defined as the direction $x$ decreases, and vice versa. After performing Fourier transform on the global time $t_{g}$ and getting the corresponding frequency $\omega$, the equations become

$$
\begin{aligned}
& \omega \psi_{+}(\omega, x)+i \partial_{x} \psi_{+}(\omega, x)=-m \psi_{-}(\omega, x) \csc (x), \\
& \omega \psi_{-}(\omega, x)-i \partial_{x} \psi_{-}(\omega, x)=-m \psi_{+}(\omega, x) \csc (x) .
\end{aligned}
$$

Next we abbreviate $\psi_{-/+}(\omega, x)$ as $\psi_{-/+}(x)$ for simplicity. This set of differential equations can be analytically solved. By introducing the new variable $\psi_{1}(x)=\psi_{+}(x)+i \psi_{-}(x)$ and $\psi_{2}(x)=\psi_{+}(x)-i \psi_{-}(x)$ (here we use bold indexes 1 and $\mathbf{2}$ to avoid possible confusion with the label on the Keldysh contour), the solution of (A2) reads [44]

$$
\begin{aligned}
\psi_{\mathbf{1}}(x)= & \frac{(1+\cos (x))^{\frac{1}{4}-\frac{m}{2}}}{\sqrt{\sin (x)}}\left[\tilde{C}_{1}(1-\cos (x))^{\frac{1}{4}+\frac{m}{2}}{ }_{2} F_{1}\left(-\omega, \omega ; m+\frac{1}{2} ; \sin ^{2}\left(\frac{x}{2}\right)\right)\right. \\
& \left.+\tilde{C}_{2} \frac{i 2^{\frac{1}{2}+m} \omega}{1-2 m}(1-\cos (x))^{\frac{3}{4}-\frac{m}{2}}{ }_{2} F_{1}\left(-m-\omega+\frac{1}{2},-m+\omega+\frac{1}{2} ; \frac{3}{2}-m ; \sin ^{2}\left(\frac{x}{2}\right)\right)\right], \\
\psi_{\mathbf{2}}(x)= & \frac{(1+\cos (x))^{\frac{1}{4}+\frac{m}{2}}}{\sqrt{\sin (x)}}\left[\tilde{C}_{1}(1-\cos (x))^{\frac{1}{4}-\frac{m}{2}}{ }_{2} F_{1}\left(-\omega, \omega ; \frac{1}{2}-m ; \sin ^{2}\left(\frac{x}{2}\right)\right)\right. \\
& \left.+\tilde{C}_{2} \frac{i 2^{\frac{1}{2}-m} \omega}{1+2 m}(1-\cos (x))^{\frac{3}{4}+\frac{m}{2}}{ }_{2} F_{1}\left(\frac{1}{2}+m-\omega, \frac{1}{2}+m+\omega ; \frac{3}{2}+m ; \sin ^{2}\left(\frac{x}{2}\right)\right)\right],
\end{aligned}
$$

where ${ }_{2} F_{1}(a, b ; c ; y)$ is the standard hypergeometric function and we have two undetermined constants, $\tilde{C}_{1}$ and $\tilde{C}_{2}$.

We then consider the black-hole geometry with metric $d s^{2}=-r(r-2) d t_{R}^{2}+d r^{2} / r(r-2)$. Now we define the $\Psi=$ $\left(\psi_{-}, \psi_{+}\right)$, which gives

$$
\begin{gathered}
-\frac{\partial_{t_{R}} \psi_{-}\left(t_{R}, r\right)}{\sqrt{r(r-2)}}+\sqrt{r(r-2)} \partial_{r} \psi_{-}\left(t_{R}, r\right)+\frac{r-1}{2 \sqrt{r(r-2)}} \psi_{-}\left(t_{R}, r\right)+m \psi_{+}\left(t_{R}, r\right)=0, \\
\frac{\partial_{t_{R}} \psi_{+}\left(t_{R}, r\right)}{\sqrt{r(r-2)}}+\sqrt{r(r-2)} \partial_{r} \psi_{+}\left(t_{R}, r\right)+\frac{r-1}{2 \sqrt{r(r-2)}} \psi_{+}\left(t_{R}, r\right)+m \psi_{-}\left(t_{R}, r\right)=0,
\end{gathered}
$$

The bulk wave function moving to the direction where $r$ increases when $m=0$ is labeled by $\psi_{+}\left(t_{R}, r\right)$, and vice versa. Then we perform Fourier transform on the Rindler time $t_{R}$ and get the corresponding frequency $\omega$. The equation becomes

$$
\begin{gathered}
\frac{i \omega \psi_{-}(r)}{\sqrt{r(r-2)}}+\sqrt{r(r-2)} \partial_{r} \psi_{-}(r)+\frac{r-1}{2 \sqrt{r(r-2)}} \psi_{-}(r)+m \psi_{+}(r)=0, \\
-\frac{i \omega \psi_{+}(r)}{\sqrt{r(r-2)}}+\sqrt{r(r-2)} \partial_{r} \psi_{+}(r)+\frac{r-1}{2 \sqrt{r(r-2)}} \psi_{+}(r)+m \psi_{-}(r)=0 .
\end{gathered}
$$


Here we also abbreviate $\psi_{-/+}(\omega, r)$ as $\psi_{-/+}(r)$. The solutions of these differential equations have the form [13]

$$
\begin{aligned}
\psi_{-}(r)= & \left(C_{1} \frac{2^{m}\left(\frac{1}{r}\right)^{m} \boldsymbol{\Gamma}(m+1)\left(1-\frac{2}{r}\right)^{-\frac{i \omega}{2}} \boldsymbol{\Gamma}\left(m+i \omega+\frac{1}{2}\right){ }_{2} F_{1}\left(m, m-i \omega+\frac{1}{2} ; 2 m+1 ; \frac{2}{r}\right)}{\left(r^{2}-2 r\right)^{1 / 4} \boldsymbol{\Gamma}(2 m+1) \boldsymbol{\Gamma}\left(i \omega+\frac{1}{2}\right)}\right. \\
& \left.+C_{2} \frac{2^{-m}\left(\frac{1}{r}\right)^{-m} \boldsymbol{\Gamma}(1-m)\left(1-\frac{2}{r}\right)^{-\frac{i \omega}{2}} \boldsymbol{\Gamma}\left(-m+i \omega+\frac{1}{2}\right){ }_{2} F_{1}\left(-m,-m-i \omega+\frac{1}{2} ; 1-2 m ; \frac{2}{r}\right)}{\left(r^{2}-2 r\right)^{1 / 4} \boldsymbol{\Gamma}(1-2 m) \boldsymbol{\Gamma}\left(i \omega+\frac{1}{2}\right)}\right), \\
\psi_{+}(r)= & \left(C_{1} \frac{2^{m}\left(\frac{1}{r}\right)^{m} \boldsymbol{\Gamma}(m+1)\left(1-\frac{2}{r}\right)^{\frac{i \omega}{2}} \boldsymbol{\Gamma}\left(m+i \omega+\frac{1}{2}\right){ }_{2} F_{1}\left(m, m+i \omega+\frac{1}{2} ; 2 m+1 ; \frac{2}{r}\right)}{\left(r^{2}-2 r\right)^{1 / 4} \boldsymbol{\Gamma}(2 m+1) \boldsymbol{\Gamma}\left(i \omega+\frac{1}{2}\right)}\right. \\
& \left.-C_{2} \frac{2^{-m}\left(\frac{1}{r}\right)^{-m} \boldsymbol{\Gamma}(1-m)\left(1-\frac{2}{r}\right)^{\frac{i \omega}{2}} \boldsymbol{\Gamma}\left(-m+i \omega+\frac{1}{2}\right){ }_{2} F_{1}\left(-m,-m+i \omega+\frac{1}{2} ; 1-2 m ; \frac{2}{r}\right)}{\left(r^{2}-2 r\right)^{1 / 4} \boldsymbol{\Gamma}(1-2 m) \boldsymbol{\Gamma}\left(i \omega+\frac{1}{2}\right)}\right) .
\end{aligned}
$$

Here we have two undetermined constants, $C_{1}$ and $C_{2}$.

[1] S. Hartnoll, A. Lucas, and S. Sachdev, Holographic Quantum Matter (MIT Press, Cambridge, MA, 2018).

[2] A. Y. Kitaev, Talks at KITP, University of California Santa Barbara, (unpublished), http://online.kitp.ucsb.edu/ online/entangled15/kitaev/, http://online.kitp.ucsb.edu/online/ entangled15/kitaev2/.

[3] S. Sachdev and J. Ye, Phys. Rev. Lett. 70, 3339 (1993).

[4] J. Maldacena and D. Stanford, Phys. Rev. D 94, 106002 (2016).

[5] A. Kitaev and S. J. Suh, J. High Energy Phys. 05 (2018) 183.

[6] J. Maldacena, D. Stanford, and Z. Yang, Prog. Theor. Exp. Phys. 2016, 12 C104 (2016).

[7] N. V. Gnezdilov, J. A. Hutasoit, and C. W. J. Beenakker, Phys. Rev. B 98, 081413(R) (2018).

[8] A. Kruchkov, A. A. Patel, P. Kim, and S. Sachdev, Phys. Rev. B 101, 205148 (2020).

[9] A. Altland, D. Bagrets, and A. Kamenev, Phys. Rev. Lett. 123, 226801 (2019).

[10] O. Can, E. M. Nica, and M. Franz, Phys. Rev. B 99, 045419 (2019).

[11] S. Sachdev, Phys. Rev. X 5, 041025 (2015).

[12] R. A. Davison, W. Fu, A. Georges, Y. Gu, K. Jensen, and S. Sachdev, Phys. Rev. B 95, 155131 (2017).

[13] Y. Gu, A. Kitaev, S. Sachdev, and G. Tarnopolsky, J. High Energy Phys. 02 (2020) 157.

[14] J. Maldacena and X.-L. Qi, arXiv:1804.00491.

[15] S. Banerjee and E. Altman, Phys. Rev. B 95, 134302 (2017).

[16] X. Chen, R. Fan, Y. Chen, H. Zhai, and P. Zhang, Phys. Rev. Lett. 119, 207603 (2017).

[17] P. Zhang, Phys. Rev. B 96, 205138 (2017).

[18] S.-K. Jian and H. Yao, Phys. Rev. Lett. 119, 206602 (2017).

[19] Y. Gu, X.-L. Qi, and D. Stanford, J. High Energy Phys. 05 (2017) 125.

[20] D. V. Khveshchenko, Lith. J. Phys. 60, 185 (2020).

[21] D. V. Khveshchenko, Condens. Matter 5, 37 (2020).

[22] I. R. Klebanov, A. Milekhin, G. Tarnopolsky, and W. Zhao, arXiv:2006.07317.

[23] L. García-Álvarez, I. L. Egusquiza, L. Lamata, A. Del Campo, J. Sonner, and E. Solano, Phys. Rev. Lett. 119, 040501 (2017).
[24] Z. Luo, Y.-Z. You, J. Li, C.-M. Jian, D. Lu, C. Xu, B. Zeng, and R. Laflamme, npj Quantum Info. 5, 1 (2019).

[25] I. Danshita, M. Hanada, and M. Tezuka, Prog. Theor. Exp. Phys. 2017, 083 I01 (2017).

[26] A. Chen, R. Ilan, F. de Juan, D. I. Pikulin, and M. Franz, Phys. Rev. Lett. 121, 036403 (2018).

[27] C. Wei and T. A. Sedrakyan, arXiv:2005.07640.

[28] A. Chew, A. Essin, and J. Alicea, Phys. Rev. B 96, 121119(R) (2017).

[29] P. Gao, D. L. Jafferis, and A. C. Wall, J. High Energy Phys. 12 (2017) 151.

[30] J. Maldacena, D. Stanford, and Z. Yang, Fortschr. Phys. 65, 1700034 (2017).

[31] P. Saad, S. H. Shenker, and D. Stanford, arXiv:1806.06840.

[32] A. Almheiri, T. Hartman, J. Maldacena, E. Shaghoulian, and A. Tajdini, J. High Energy Phys. 05 (2020) 013.

[33] G. Penington, S. H. Shenker, D. Stanford, and Z. Yang, arXiv:1911.11977.

[34] S. Plugge, É. Lantagne-Hurtubise, and M. Franz, Phys. Rev. Lett. 124, 221601 (2020).

[35] X.-L. Qi and P. Zhang, J. High Energy Phys. 05 (2020) 129.

[36] J. Maldacena and A. Milekhin, arXiv:1912.03276.

[37] S. Sahoo, E. Lantagne-Hurtubise, S. Plugge, and M. Franz, Phys. Rev. Res. 2, 043049 (2020).

[38] N. Sorokhaibam, arXiv:2007.07169.

[39] T.-G. Zhou, L. Pan, Y. Chen, P. Zhang, and H. Zhai, arXiv:2009.00277.

[40] É. Lantagne-Hurtubise, S. Plugge, O. Can, and M. Franz, Phys. Rev. Res. 2, 013254 (2020).

[41] S.-K. Jian, Z.-Y. Xian, and H. Yao, Phys. Rev. B 97, 205141 (2018).

[42] Y. Chen, X.-L. Qi, and P. Zhang, J. High Energy Phys. 06 (2020) 121.

[43] Y. Chen and P. Zhang, J. High Energy Phys. 07 (2019) 033.

[44] We acknowledge that the solutions of (A2) were obtained by Yiming Chen. 